\title{
Reactivation of an appetitive discrimination memory following retroactive interference
}

\author{
GREGORY J. SMITH and NORMAN E. SPEAR \\ State University of New York, Binghamton, New York 13901
}

\begin{abstract}
The present experiment employed an appetitive spatial discrimination response to examine alleviation of forgetting induced by retroactive interference. For separate groups of rats, a footshock that had occurred during acquisition contingent upon a correct response was tested for its effectiveness as a reactivation treatment. The results indicated that the footshock effectively alleviated forgetting, but only for rats experiencing the shock stimulus during original learning. These results suggest that a reactivation treatment may arouse affective or stimulus properties in common with the target memory to elicit retrieval of that memory.
\end{abstract}

In the past few years, the study of memory retrieval mechanisms in animals has centered on the effectiveness of "reinstatement" or "reactivation" treatments in alleviating forgetting (Campbell \& Jaynes, 1966; Lewis, 1976; Spear, 1973, 1976; Spear \& Parsons, 1976). These treatments normally consist of reexposing the organism to some aspect of the original training situation without providing a complete training trial. The effectiveness of these treatments in the elicitation and retrieval of the target memory is believed to depend on their arousal of a sufficient number or kind of the existing, but otherwise inaccessible, attributes of that memory (Spear, 1978, chap. 2). It has been argued, however, that since the most widely used and also the most effective reactivation agent is the unconditioned stimulus (either a footshock or the appetitive reinforcer), these treatments may provide an additional learning experience which adds to the retention performance of the animal (Gold, Haycock, Macri, \& McGaugh, 1973; for another view, see Schneider, 1974). It is, of course, impossible to deny the existence of some weak reinforcement effects due to a reactivation treatment, and these effects might conceivably act upon some extratask responses. But even so, the consequences of such an effect on the index of retention are most certainly insignificant, since controls receiving only the reactivation treatments have not shown any reliable "new" learning (Spear, 1973).

While the above explanation appears adequate to discount new learning as a major contributor to the effectiveness of a reactivation treatment, it remains

Preparation of this articie was supported by grants from the National Science Foundation (BMS 74-24194 and BNS 7802360) to the second author. Thanks to Norman Richter and Jonathan Gibralter for technical advice and assistance and Teri Tanenhaus for preparation of the manuscript. Requests for reprints should be sent to Dr. Norman E. Spear, Department of Psychology, State University of New York at Binghamton, Binghamton, New York 13901. important to establish reactivation treatments that have minimal similarity with the requirements of the retention test. Toward this end, we designed the present experiment to investigate the effectiveness of a reactivation treatment in alleviating forgetting of an appetitive spatial discrimination in a T-maze. Previous studies involving appetitive memories have found the reinforcer (Miller, Ott, Berk, \& Springer, 1974), the reinforcer and instrumental response (Hamberg \& Spear, 1978), and reinforcer and discriminative stimuli (Campbell \& Randall, 1976) to be effective reactivation agents.

In the present experiment, the reactivation treatment employed included neither a discriminative stimulus nor a reinforcer, to avoid the "new learning" possibility noted above. Using a training procedure similar to that used by Fowler and Wischner (1969), rats were trained on a position discrimination in a T-maze. Upon making a correct choice, they were presented with a weak footshock. The choice of pairing shock with the correct alternative of the T-maze (and food reward) as opposed to the incorrect alternative (and nonreward) was based on several prior studies which had indicated only the former to be an effective reactivation treatment (Smith, Gibralter, \& Leight, Note 1; Gibralter, Devlin, \& Spear, Note 2). Twenty-four hours after original learning, rats in selected groups experienced a source of retroactive interference with no shock present. Twenty-four hours or 7 days later, rats were reexposed to only the shock, as the reactivation treatment, just prior to the retention test.

This present procedure affords two major advantages over previous experiments. (1) Due to the use of a two-choice spatial discrimination, the effectiveness of the reactivation treatment can be better separated from performance artifacts which may enter into the testing situation. (2) Since the reactivation treatment is removed from the apparatus and reinforcement contingencies (e.g., the food reward) 
present during testing, this eliminates the possibility that one choice of the T-maze will be incremented over another due to new learning.

\section{METHOD}

\section{Subjects}

The subjects were 84 female albino rats, approximately 60 days old, of the Sprague-Dawley strain, born and raised in our colony at the State University of New York at Binghamton. Throughout the experiment, all rats were individually housed in laboratory steel mesh cages and maintained on a 0700 (lights on) - $2300 \mathrm{~h}$ (lights off) cycle. All training and testing took place between 1100 and $1600 \mathrm{~h}$.

\section{Apparatus}

The apparatus was a T-maze (stem, $36 \mathrm{~cm}$ long $\times 11 \mathrm{~cm}$ wide $\times 12 \mathrm{~cm}$ high; each arm, $39 \mathrm{~cm}$ long $\times 16 \mathrm{~cm}$ wide $\times$ $12 \mathrm{~cm}$ high) made of clear Plexiglas and mounted on a grid floor (.4-cm-diam grid bars spaced $1.5 \mathrm{~cm}$ apart). The grid floor could be electrified by a Lehigh Valley Electronics shock generator and scrambler (Model 26861) with power supplied by a transformer from the Scientific Prototype Manufacturing Co. (Model $4026 \mathrm{~J}$ ). The stem, choice section, and arms were separated by clear Plexiglas guillotine doors. The maze was constantly illuminated from below by four $6-\mathrm{W}$ incandescent bulbs.

The reactivation chamber was made of clear Plexiglas, and it measured $33 \mathrm{~cm}$ long $\times 30 \mathrm{~cm}$ wide $\times 64 \mathrm{~cm}$ high. The chamber was equipped with a grid floor, identical to the T-maze, which could be attached to the same shock source.

\section{Pretreatment Procedure}

All animals were individually housed and weighed for a 3-day free-feeding baseline period before being gradually reduced to $80 \%$ of their baseline body weights. After reaching $80 \%$ ad-lib body weight, on each of the next 7 days each rat was weighed and then placed in a wooden box inside the experimental room for $3 \mathrm{~min}$ where it was allowed to eat 10 Noyes food pellets. At the end of this 7-day familiarization period, training began.

\section{Training Procedure}

The rats learned a position discrimination in a $\mathbf{T}$-maze to obtain three $45-\mathrm{mg}$ Noyes food pellets. All rats were randomly assigned a correct choice (left or right) and given 20 trials each day until the training criterion $(9$ correct choices in 10 consecutive free-choice trials) was met. For the purpose of training, an alternating free-choice, forced-choice, noncorrection procedure was used. This meant that on all odd-number trials $(1,3,5, \ldots$ $17,19)$, the rat was allowed to choose between both arms of the T-maze, whereas on all even-number trials, the rat was forced to enter the arm not visited on the previous free-choice trial. With this procedure, the amount of experience with each alternative was held constant. Following completion of each trial, the rat was returned to a holding cage for an intertrial interval of $30 \mathrm{sec}$. This basic procedure was followed for both original learning and reversal learning.

Original learning. For the first six training trials on Day 1, each rat was trained with the procedure outlined above, in the absence of any experimental treatments (see below). Starting with the seventh trial, the experimental treatment was introduced and continued throughout original learning until the learning criterion was met. The following experimental treatments were administered during original learning. (1) The shock correct group (SC) received a .4-mA footshock for $.4 \mathrm{sec}$ immediately upon making a correct choice response. (2) The no-treatment group (NT) received no additional cues beyond those available on Trials 1-6. (3) The reversal only group ( $R$ ) received no training during this stage. In each case, a choice was defined as the placement of all four paws inside an arm of the T-maze. Latency measurements consisted of time (recorded to hundredths of a second) required to leave the startbox and make a choice response. Upon reaching the learning criterion, each rat was returned to its home cage for $24 \mathrm{~h}$ before reversal learning.

Reversal training. At no time during this stage was a shock trial administered. At this point, Group $\mathbf{R}$ was given its only training experience. For all other groups, the reversal-learning stage served as a source of retroactive interference, with no food present in the alternative formerly rewarded during original learning. Each animal received a maximum of 20 trials per day (10 free-choice and 10 forced-choice trials) with the same training schedule used during original learning. Upon reaching criterion ( 8 correct choices in 10 consecutive free-choice trials), each rat was returned to its home cage until testing. Testing occurred 24 h or 7 days after attainment of the reversal criterion.

\section{Reactivation/No-Reactivation Treatment}

Prior to testing, each group was divided randomly into two treatment groups. One group received reactivation treatment and the other received no reactivation treatment. Reactivation treatment consisted of two .55-sec .4-mA footshocks presented in a clear Plexiglas apparatus $10 \mathrm{~min}$ prior to testing. The footshocks were separated by $30 \mathrm{sec}$., which the rat spent in a holding cage. After the reactivation treatment, the rat was returned to its home cage until testing. Those animals receiving no reactivation treatment were handled, carried to the experimental room, and returned to their home cage without being placed in the reactivation apparatus.

\section{Testing Procedure}

Testing occurred $10 \mathrm{~min}$ after the reactivation or no-reactivation treatments. Testing consisted of 10 free-choice trials in the same apparatus used for training. Unlike training, testing involved no forced-trials and both arms of the maze were baited with the food pellets.

\section{RESULTS}

\section{Acquisition}

Original learning. A 2 by 2 analysis of variance ( 2 treatment conditions, Groups SC and NT by reactivation vs. no reactivation) was performed on the number of trials required by each rat to reach the original learning criterion ( 9 correct choices in any 10 consecutive free-choice trials). This analysis indicated that treatment condition $[F(1,52)=3.76$, p $>.05]$, reactivation vs. no reactivation $(F<1)$, and their interaction $[F(1,52)=2.06, p>.10]$ were insignificant sources of variation. The mean total number of trials required to reach the learning criterion were 23.5 and 20.0 for Groups SC and NT, respectively.

In order to determine whether animals in each group had learned the discrimination equally well, the latency scores were partitioned by reinforced (correct) and nonreinforced (incorrect) trials for each animal over the last 5 correct choice trials and the last 5 incorrect choice trials. This determination is especially important for the present procedure. It is possible that our treatment groups had an advantage over our untreated groups in learning through the presence of additional "cues"- the shock that occurred following a choice. On the other hand, to ascribe unique motivational properties to those cues 
paired with the choice response, it should be shown that their presentation resulted in either an increase in rate of learning the discrimination or an increase in running speed to the correct choice. As mentioned above, rate of learning the discrimination did not differ between groups, thereby eliminating the first alternative.

A 2 by 2 by 5 analysis of variance (Group SC vs. Group NT by latency on a correct choice vs. that on an incorrect choice by the last 5 training trials) was performed on running speed scores to the correct and incorrect arms over the last 10 trials of acquisition (5 to the correct, 5 to the incorrect arm). Analysis indicated that animals ran faster to the reinforced arm than to the nonreinforced arm $[F(1,54)=39.41, p<.001)$. No other main effects or interactions were significant. This analysis further demonstrates that Group SC was not afforded a reliable advantage over Group NT in learning the spatial discrimination due to the addition of the shock cue during original learning.

Reversal learning. A 3 by 2 analysis of variance (3 treatment groups, Groups SC, NT, R, by reactivation vs. no reactivation) performed on the number of trials required by each rat to reach the reversal criterion indicated no reliable interaction $(F<1)$. Similarly, no significant main effects were indicated between treatment groups $[F(2,78)=1.83, p>.10]$ or between reactivation/no reactivation groups $[F(1,78)=1.44, p>.10]$. The mean numbers of free-choice trials to reach the learning criterion for Groups SC, R, NT were 12.4 , 10.8, and 13.4, respectively. In spite of the trend toward negative transfer of original learning to reversal learning observed in Groups SC and NT, these differences were not found to be reliable in our analysis. These results add further support that the original learned response was acquired to an equivalent degree for all treatment groups, allowing us to assess subsequent retention unconfounded by these factors.

\section{Retention Test}

The probability of choosing the alternative rewarded during original learning is reported in Figure 1. As can be seen from the figure, the reactivation treatments alleviated the forgetting induced by retroactive interference (reversal learning) in only the group SC, irrespective of the length of the retention interval. No similar effects were observed in either the NT or R groups. A 2 by 3 by 2 analysis of variance $(2$ retention intervals by 3 treatment groups by reactivation vs. no reactivation) performed on the individual test scores (probability of choosing the arm rewarded during original learning) confirmed this interpretation. The analysis indicated a reliable two-way interaction between the treatment groups (SC, NT, R) and the presence or absence of the reactivation treatments $[F(2,72)=26.87, p<.001]$. The analysis further indicated reliable main effects of treatment conditions $[F(2,72)=21.22, p<.001]$ and presence of a reactivation treatment $[F(1,72)=8.13, p<.01]$. No other interactions or main effects were statistically significant. The major interest of this experiment was in examining the effectiveness of the reactivation treatment in alleviating forgetting due to retroactive interference. To do this, planned comparisons were calculated separately for groups tested after $24 \mathrm{~h}$ and for those tested after 7 days, using the error term from the factorial analysis.

24-Hour retention test. The results of the 24-h retention test are represented in the left panel of Figure 1. Simple retention of the spatial discrimination is best indexed by the retention scores of the nonreactivated rats in Group R. An analysis of the retention scores (probability of originally correct choice) of nonreactivated rats in Groups NT and SC compared to Group $\mathbf{R}$ indicated that none of these scores differed reliably from another at the .05 level. That Group $\mathrm{R}$ showed no better retention than the other two nonreactivated groups is consistent with other experiments which have failed to find proactive interference in the retention of a simple spatial discrimination (see Spear, 1971, for a review). Further, that Groups NT and SC showed no better retention of the original discrimination than Group $\mathrm{R}$ (which never had learned that discrimination) suggests that substantial retroactive interference, provided by interpolated reversal learning, was manifested by the nonreactivated groups at the 24-h retention test.

Comparison between the reactivated and nonreactivated groups within each treatment condition

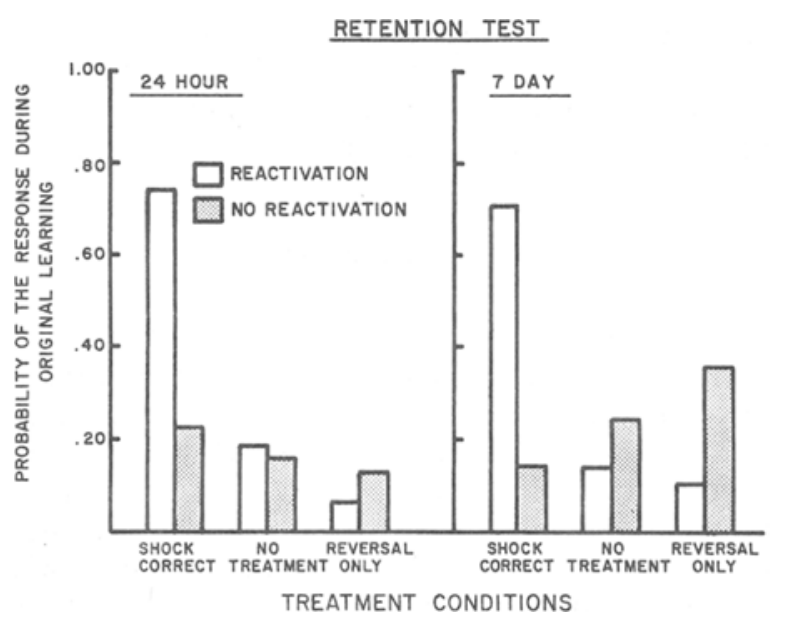

Figure 1. The mean probability of choosing the arm of the maze previously reinforced during original learning. Note: Since Group $R$ had not experienced original learning, their score represents the probability of an error on test. 
assesses the effectiveness of the reactivation treatment in alleviating the forgetting caused by the source of retroactive interference. Presentation of the (shock) reactivation treatment to rats given the shock during original learning (Group SC) increased the probability of choosing the response alternative rewarded during original learning relative to the nonreactivated SC animals. Planned comparisons indicated that this alleviation of forgetting following the reactivation treatment was highly reliable $[F(1,36)=9.39, p<.01$; see Figure 1, left panel]. Neither Group NT nor Group R exhibited a similar improvement following the reactivation treatment (both, $F<1$ ).

7-Day retention test. The retention test results 7 days after reversal learning are reported in the right panel of Figure 1. Planned comparisons performed on the treatment groups indicated that the reactivation treatment reliably improved the retention of original learning for only Group $\operatorname{SC}[F(1,36)=9.68$, $\mathrm{p}<.01]$. None of the other groups exhibited a similar benefit from the reactivation treatment. For Group NT, retention by the nonreactivated rats was not reliably different from that of the reactivated rats $(\mathrm{F}<1)$.

Finally, although the trend was suggestive, simple retention of the spatial discrimination 7 days after learning was not reliably influenced by the presence of the reactivation treatment in Group $R$. The probability of an error for nonreactivated rats was not statistically different from that for the reactivated rats $[\mathrm{F}(1,36)=1.85, \mathrm{p}<.10]$.

Analysis of response latency on the first test trial led to the same conclusions as that for choice behavior. Briefly, a 2 by 3 by 2 analysis of these latencies indicated a reliable Treatment Group by Reactivation/No Reactivation interaction $[F(2,72)=$ $4.04, \mathrm{p}<.05$; no other interactions or main effects were significant]. Reactivated SC rats ran faster (mean $=3.6 \mathrm{sec})$ on the first test trial than did nonreactivated SC rats (mean $=9.0 \mathrm{sec}$ ), while the reactivation treatment did not affect behavior in the remaining groups.

\section{DISCUSSION}

The present experiment examined the effectiveness of a reactivation treatment in alleviating forgetting of a spatial discrimination induced by retroactive interference. The results indicated that (1) under certain circumstances, an apparently redundant training stimulus, associated with the correct response alternative in a T-maze, will elicit retrieval of an otherwise inaccessible memory; (2) the effectiveness of the reactivation treatment appears due to an increase in the accessibility of the target memory, as opposed to providing an additional new learning trial that adds to the existing residual memory. Had the reactivation treatment acted as a new learning trial, it would be difficult to explain how it differentially influenced preference for one alternative of the T-maze over another, and then only for rats experiencing the redundant training stimulus during original learning.

It is our belief that this pretest exposure to the redundant training stimulus (footshock), provided by the sensory detection and perception of the footshock and its aftereffects, increased the contextual similarity between the testing environment and that of original learning. The greater the similarity between training and testing, the better performance will be on the retention test (Spear, 1978, Chap. 2). In the present experiment, because the reinforcer (food pellets) was present during original learning only after a brief inescapable footshock, the attributes of approach and consumption of food may have become conditioned to the sensory consequences of the footshock and the relatively persistent aftereffects of the footshocks-probably hormonal and internal consequences rather than somesthetic in nature-that formed an important part of the experimental context. When the noncontingent footshock was presented prior to testing, the resulting internal and external consequences of the shock made the contextual environment of testing more similar to that present during original learning than to that during reversal learning. Accessibility of the target memory associated with original learning was thereby increased for rats experiencing the redundant shock stimulus during original learning. Note that the shock failed to have an effect on rats not experiencing the shock stimulus during original learning. Hence it appears that this reactivation treatment must have aroused common affective or stimulus properties with the target memory in order to elicit retrieval of that memory.

Finally, a number of experiments have demonstrated that mild-shock punishment may act to increase the discriminability of a response and facilitate the rate of acquisition of a visual $T$-maze discrimination (Fowler, 1971; Fowler \& Wischner, 1969; Muenzinger, 1934). That the present experiments did not demonstrate this facilitation effect is not unexpected, however, in light of the experimental parameters used. For instance, Fowler has indicated that this facilitation effect is dependent on the difficulty of the discrimination and often requires training rats to a very stringent criterion. Furthermore, cue facilitation of learning has typically not been found in easy discriminations where the stimulus alternatives were highly discriminable (i.e., simple spatial discrimination). This agrees with Sutherland and Mackintosh (1971), who have argued that the more difficult the discrimination, the more the organism learns about the redundant cues and therefore the more likely they are to benefit from the use of redundant 
cues. The present experiment indicates that while the rat may not come to benefit from the use of the redundant cue in acquiring the response, this cue does make up an important part of the experimental situation and may be used to facilitate retrieval of the memory.

\section{REFERENCE NOTES}

1. Smith, G. J., Gibralter, J., \& Leight, K. Cue-specific reactivation of an appetitive task. Paper presented at the meeting of the Midwestern Psychological Association, Chicago, 1977.

2. Gibralter, J., Devlin, L., \& Spear, N. E. Reactivation of a discrimination memory with redundant olfactory cues. Paper presented at the 13th Annual Undergraduate Convention at Hobart and William Smith College, 1978.

\section{REFERENCES}

Campbell, B. A., \& Jaynes, J. Reinstatement. Psychological Review, 1966, 73, 478-480.

Campbell, B. A., \& Randall, P. K. The effects of reinstatement stimulus conditions on the maintenance of long term memory. Developmental Psychobiology, 1976, 9, 325-334.

Fowle R, H. Suppression and facilitation by response contingent shock. In F. R. Brush (Ed.), A versive conditioning and learning. New York: Academic Press, 1971.

Fowler, H., \& WISChNer, G. J. The varied functions of punishment in discrimination learning. In B. A. Campbell \& R. A. Church (Eds.), Punishment and aversive behavior. New York: Appleton-Century-Crofts, 1969.

Gold, P. E., Haycock, J. W., Macri, J., \& McGaugh, J. L. Retrograde amnesia and the "reminder effect": An alternative interpretation. Science, 1973, 180, 1199-1201.
Hamberg, J., \& Spear, N. E. Alleviation of forgetting of discrimination learning. Learning and Motivation, 1978, 9, 466-476.

LEwIS, D. J. A cognitive approach to experimental amnesia. American Journal of Psychology, 1976, 89, 51-80.

Miller, R. R., Ott, C. A., Berk, A. M., \& Springer, A. D. Appetitive memory restoration after electroconvulsive shock in the rat. Journal of Comparative and Physiological Psychology, $1974,87,717-723$.

Muenzinger, K. F. Motivation in learning: 1. Electric shock for correct responses in the visual discrimination habit. Journal of Comparative Psychology, 1934, 17, 267-278.

Schneider, A. M. Retrograde amnesia and the "reminder effect." Science, 1974, 186, 1135-1136.

Spear, N. E. Forgetting as retrieval failure. In W. K. Honig \& P. H. R. James (Eds.), Animal memory. New York: Academic Press, 1971.

Spear, N. E. Retrieval of memory in animals. Psychological Review, 1973, 80, 163-194.

SpEAR, N. E. Retrieval of memories: A psychobiological approach. In W. K. Estes (Ed.), Handbook of learning and cognitive processes (Vol. 4) Memory processes. Hillsdale, N.J: Erlbaum, 1976.

Spear, N. E. The processing of memories: Forgetting and retention. Hillsdale, N.J: Erlbaum, 1978.

Spear, N. E., \& Parsons, P. Analysis of a reactivation treatment: Ontogeny and alleviated forgetting. In D. Medin, R. Davis, \& W. Roberts (Eds.), Coding processes in animal memory. Hillsdale, N.J: Erlbaum, 1976.

Sutherland, N. S., \& Mackintosh, N. J. Mechanisms of animal discrimination learning. London: Academic Press, 1971.

(Received for publication June 30, 1978; revision accepted January 4, 1979.) 